\title{
Weighted Version of Generalized Inverse Weibull Distribution
}

Sofi Mudiasir

University of Kashmir, Srinagar, India, sofimudasir3806@gmail.com

S.P.Ahmad

University of Kashmir, Srinagar, India

Follow this and additional works at: https://digitalcommons.wayne.edu/jmasm

Part of the Applied Statistics Commons, Social and Behavioral Sciences Commons, and the Statistical Theory Commons

\section{Recommended Citation}

Mudasir, S., \& Ahmad, S. P. (2018). Weighted version of generalized inverse Weibull distribution. Journal of Modern Applied Statistical Methods, 17(2), eP2691. doi: 10.22237/jmasm/1555506264

This Emerging Scholar is brought to you for free and open access by the Open Access Journals at DigitalCommons@WayneState. It has been accepted for inclusion in Journal of Modern Applied Statistical Methods by an authorized editor of DigitalCommons@WayneState. 


\section{EMERGING SCHOLARS}

\section{Weighted Version of Generalized Inverse Weibull Distribution}

\author{
Sofi Mudasir \\ University of Kashmir \\ Srinagar, India
}

\author{
S. P. Ahmad \\ University of Kashmir \\ Srinagar, India
}

Weighted distributions are used in many fields, such as medicine, ecology, and reliability. A weighted version of the generalized inverse Weibull distribution, known as weighted generalized inverse Weibull distribution (WGIWD), is proposed. Basic properties including mode, moments, moment generating function, skewness, kurtosis, and Shannon's entropy are studied. The usefulness of the new model was demonstrated by applying it to a real-life data set. The WGIWD fits better than its submodels, such as length biased generalized inverse Weibull (LGIW), generalized inverse Weibull (GIW), inverse Weibull (IW) and inverse exponential (IE) distributions.

Keywords: Generalized inverse Weibull distribution, weight function, Shannon's entropy, Akaike and Bayesian information criterion

\section{Introduction}

The Weibull distribution is a widely-used distribution for analyzing lifetime data. The Weibull family is a generalization of the exponential family and can model data exhibiting monotone hazard rate behavior. If the hazard function of the distribution under study is unimodal and non-monotone, then the inverse Weibull distribution can be used to analyze such data. The inverse Weibull distribution can be used to model a variety of failure characteristics such as infant mortality, useful life, and wear-out periods; see Calabria and Pulcini (1994) and Kundu and Howlader (2010). de Gusmão, Ortega, and Cordeiro (2009) proposed a threeparameter generalized inverse Weibull distribution by adding another parameter to the standard inverse Weibull distribution. Mudasir, Ahmed, and Ahmad (2015) estimated the parameters of the inverse Weibull distribution under different loss

doi: 10.22237/jmasm/1555506264 | Accepted: October 31, 2017; Published: April 17, 2019.

Correspondence: Sofi Mudasir, sofimudasir3806@gmail.com 


\section{MUDASIR \& AHMAD}

functions. Naqash, Ahmad, and Ahmed (2016) obtained the Bayes estimates of generalized inverse Weibull distribution.

The probability density function of generalized inverse Weibull distribution is given by

$$
\mathrm{f}(x)=\frac{\lambda \beta \alpha^{\beta}}{x^{\beta+1}} \exp \left(-\lambda\left(\frac{\alpha}{x}\right)^{\beta}\right) \quad x>0 ; \alpha, \beta, \lambda>0,
$$

where $\alpha$ and $\beta$ are called the scale and shape parameters, respectively.

The idea of weighted distributions was given by Fisher (1934) and studied by Rao (1965) in connection with modeling statistical data where the practice of using standard distributions for the purpose was not found to be appropriate. This may occur due to the non-observability of some events, damage caused to the original observation resulting in a reduced value, or adoption of a sampling procedure which gives unequal chances to the units in the original. Weighted distributions were used frequently in research related to reliability, bio-medicine, ecology, and branching processes can be seen in Patil and Rao (1978), Gupta and Kirmani (1990), Gupta and Keating (1985), and Oluyede (1999), and in references therein. Many researchers for weighted distributions, such as Shaban and Boudrissa (2007), have shown that the length-biased version of the Weibull distribution known as the Weibull Length-biased (WLB) distribution is unimodal throughout examining its shape, with other properties, Kersey (2010) introduced the weighted inverse Weibull distribution and beta-inverse Weibull distribution, and theoretical properties of them; Mudasir and Ahmad (2015) studied the structural properties of length biased Nakagami distribution. Fatima and Ahmad (2017) studied the weighted inverse Rayleigh distribution. Jan, Fatima, and Ahmad (2017) studied the weighted Ailamujia distribution and found its applications to real data sets.

If $X$ is a non-negative random variable with density function $\mathrm{f}(x)$ and $\mathrm{w}(x)$ is a non-negative weight function with finite non-zero expectation, then the probability density function of the weighted random variable $X_{w}$ is given by

$$
\mathrm{f}_{w}(x)=\frac{\mathrm{w}(x) \mathrm{f}(x)}{\mu_{w}}, \quad x>0,
$$

where $\mu_{w}=\mathrm{E}(\mathrm{w}(x))<\infty$.

The distribution of $X_{w}$ is known as the weighted distribution corresponding to $X$. Let 


\section{WEIGHTED GENERALIZED INVERSE WEIBULL DISTRIBUTION}

$$
\mathrm{w}(x)=x^{\theta}, \quad \theta \in \mathrm{R} .
$$

Also,

$$
\begin{aligned}
\mu_{w} & =\int_{0}^{\infty} \mathrm{w}(x) \mathrm{f}(x) d x \\
& \Rightarrow \mu_{w}=\lambda \beta \alpha^{\beta} \int_{0}^{\infty} x^{\theta-\beta-1} \exp \left(-\lambda\left(\frac{\alpha}{x}\right)^{\beta}\right) d x
\end{aligned}
$$
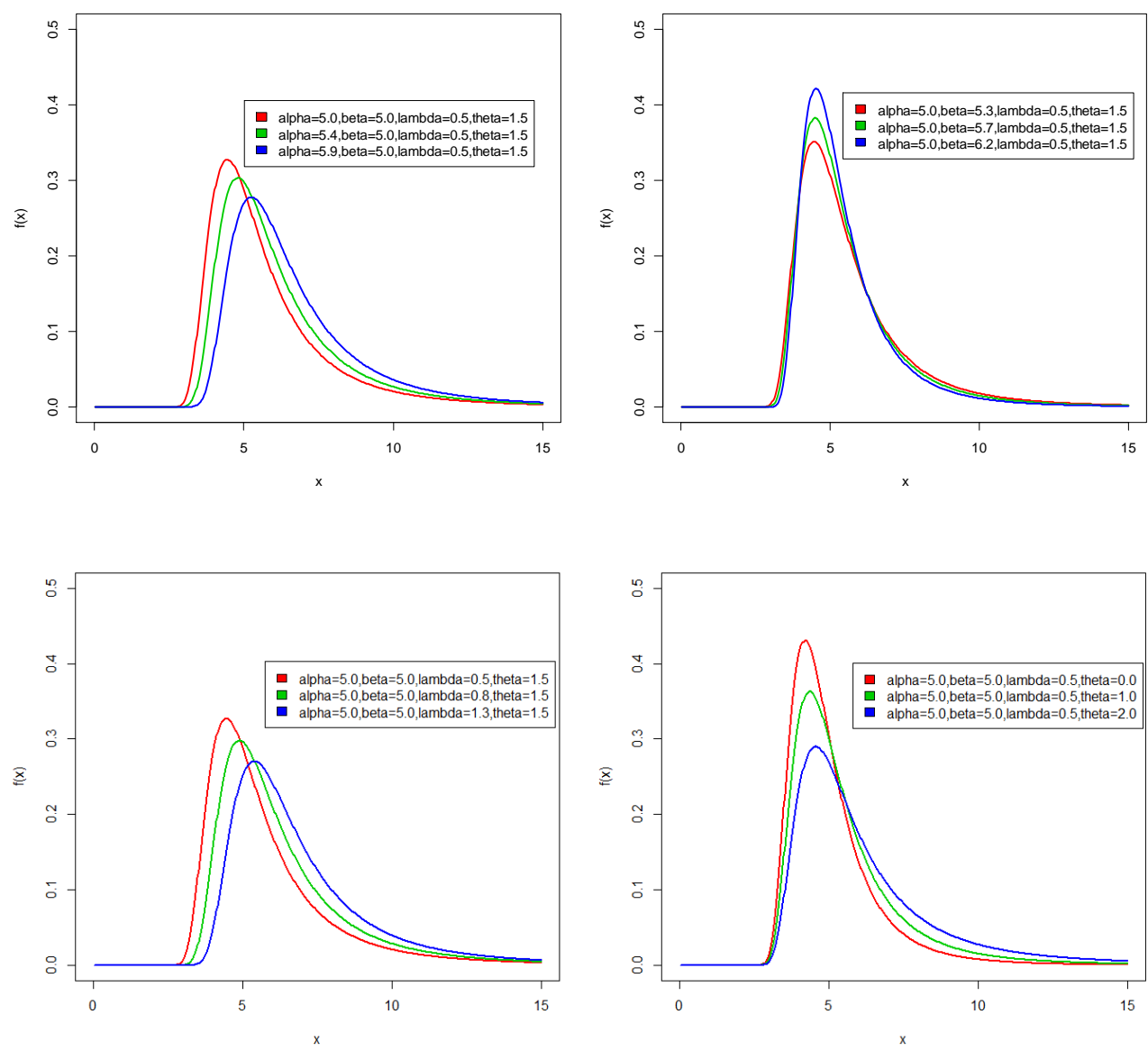

Figure 1. Probability distribution function of weighted generalized inverse Weibull distribution 


\section{MUDASIR \& AHMAD}
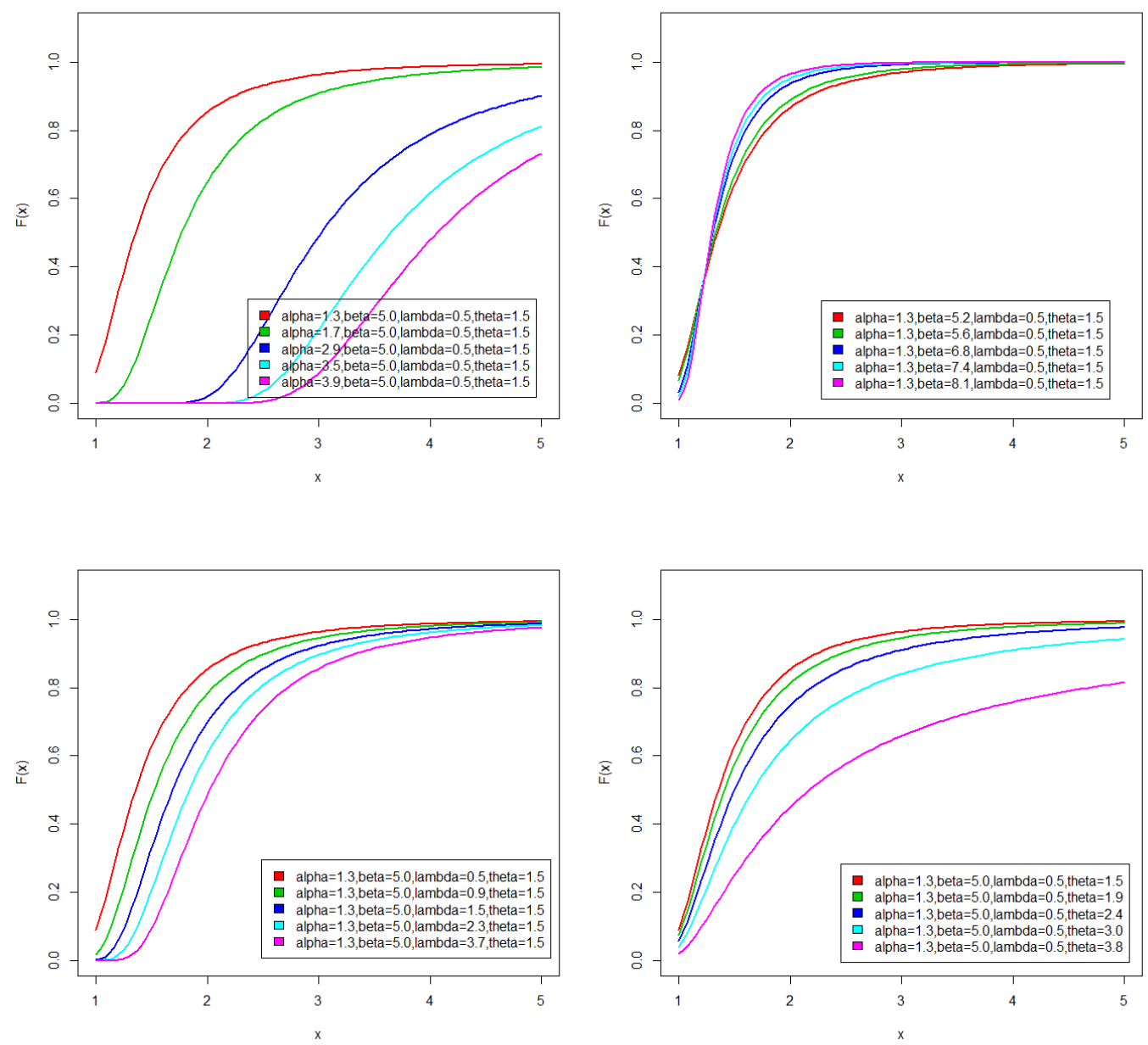

Figure 2. Cumulative distribution function of weighted generalized inverse Weibull distribution

By substituting $y=\lambda(\alpha / x)^{\beta}$,

$$
\mu_{w}=\alpha^{\theta} \lambda^{\frac{\theta}{\beta}} \Gamma\left(1-\frac{\theta}{\beta}\right)
$$

Substituting the values of equations (1), (3), and (4) into equation (2), 


\section{WEIGHTED GENERALIZED INVERSE WEIBULL DISTRIBUTION}

$$
\mathrm{f}_{w}(x)=\frac{\beta \alpha^{\beta-\theta} \lambda^{1-\frac{\theta}{\beta}} x^{\theta-\beta-1} \exp \left(-\lambda\left(\frac{\alpha}{x}\right)^{\beta}\right)}{\Gamma\left(1-\frac{\theta}{\beta}\right)}, x>0 ; \alpha, \beta, \theta, \lambda>0 .
$$

The density function in equation (5) is the weighted version of generalized inverse Weibull distribution (WGIWD).
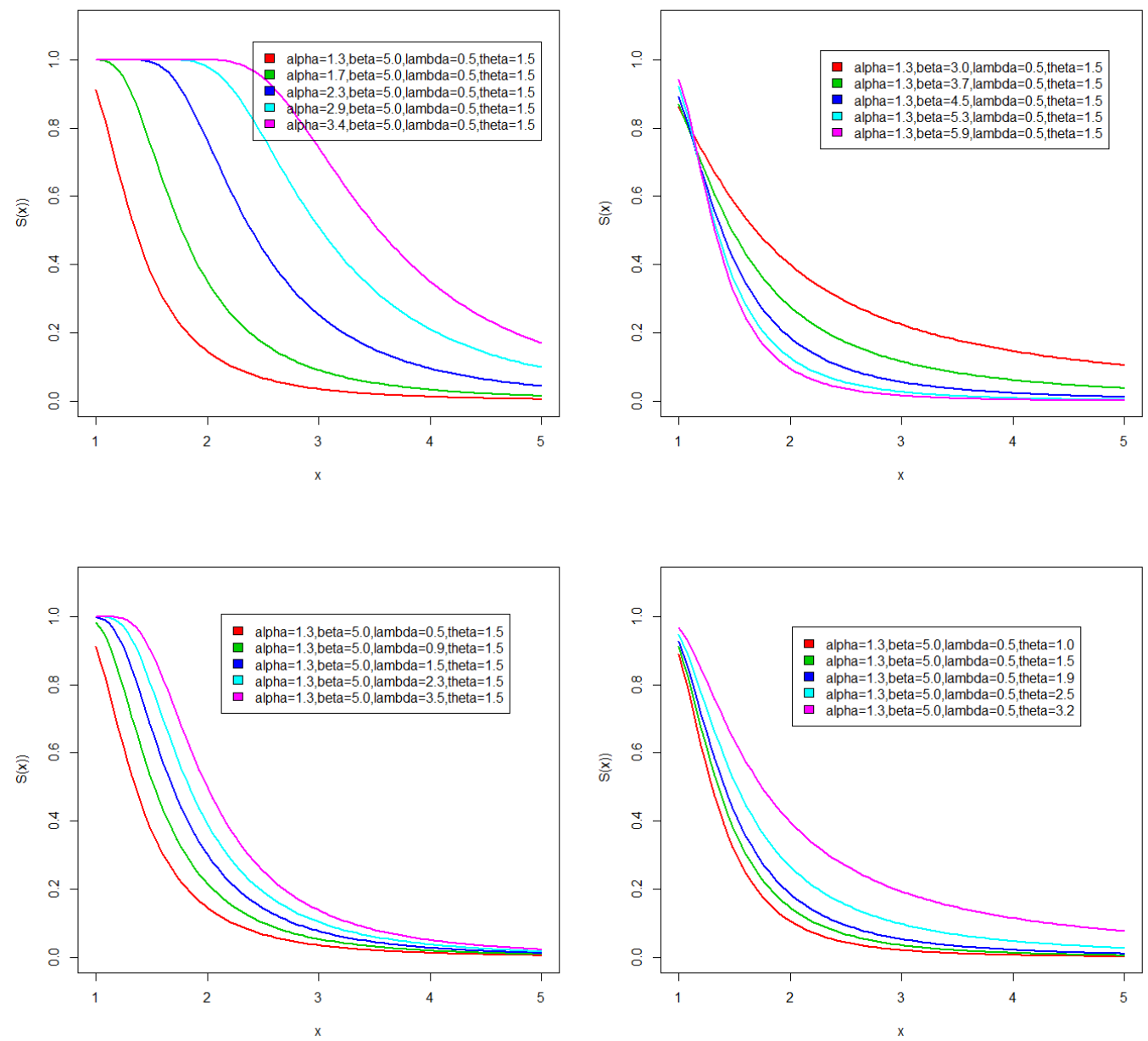

Figure 3. Survival function of the weighted generalized inverse Weibull distribution 


\section{MUDASIR \& AHMAD}
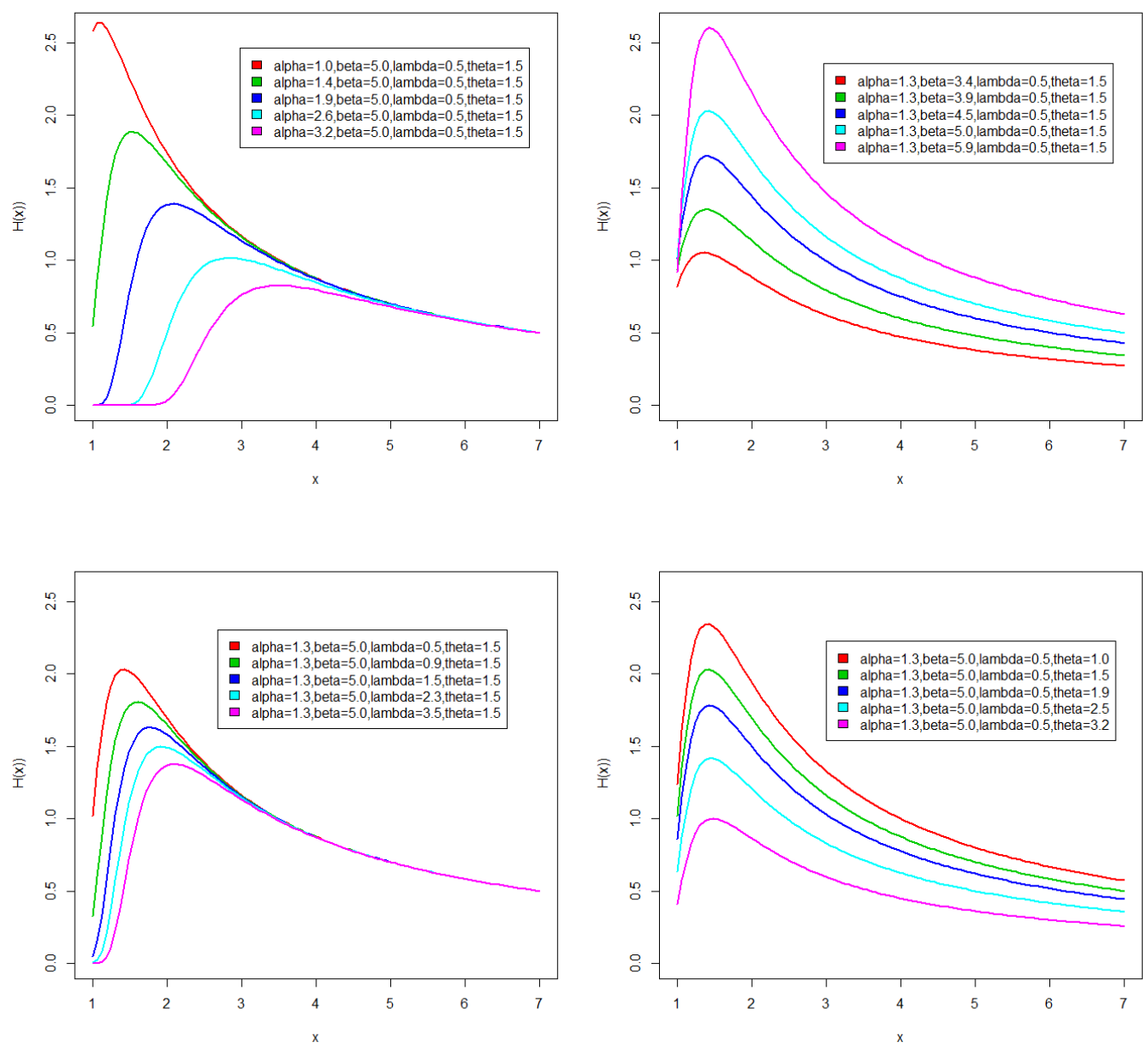

Figure 4. Hazard rate function of the weighted generalized inverse Weibull distribution

The cumulative distribution function (cdf) of (5) is defined as

$$
\mathrm{F}_{w}(x)=\frac{\Gamma\left(1-\frac{\theta}{\beta}, \frac{\lambda \alpha^{\beta}}{x^{\beta}}\right)}{\rho_{\theta}},
$$

where 


\section{WEIGHTED GENERALIZED INVERSE WEIBULL DISTRIBUTION}

$$
\rho_{\theta}=\Gamma\left(1-\frac{\theta}{\beta}\right) .
$$

The survival function of WGIWD is given by

$$
\mathrm{S}(x)=\frac{\gamma\left(1-\frac{\beta}{2}, \frac{\lambda \alpha^{\beta}}{x^{\beta}}\right)}{\rho_{\theta}} .
$$

The hazard rate function of WGIWD is given as

$$
\mathrm{h}(x)=\frac{\beta \alpha^{\beta-\theta} \lambda^{1-\frac{\theta}{\beta}} x^{\theta-\beta-1} \exp \left(-\lambda\left(\frac{\alpha}{x}\right)^{\beta}\right)}{\gamma\left(1-\frac{\beta}{2}, \frac{\lambda \alpha^{\beta}}{x^{\beta}}\right)} .
$$

\section{Some Cases of WGIWD}

1. If $\theta=1$ in (5), the length biased generalized inverse Weibull distribution is obtained with the probability density function is given as

$$
\mathrm{f}_{l}(x)=\frac{\beta \alpha^{\beta-1} \lambda^{1-\frac{1}{\beta}} x^{\beta} \exp \left(-\lambda\left(\frac{\alpha}{x}\right)^{\beta}\right)}{\rho_{\theta}}, \quad x>0 ; \alpha, \beta, \lambda>0 .
$$

2. If $\theta=0$ in (5), the generalized inverse Weibull distribution given in (1) is obtained.

3. If $\theta=0, \lambda=1$ in (5), the inverse Weibull distribution is obtained with the probability density function given as

$$
\mathrm{f}(x)=\beta \alpha^{\beta} x^{-\beta-1} \exp \left(-\left(\frac{\alpha}{x}\right)^{\beta}\right), \quad x>0 ; \alpha, \beta>0 .
$$




\section{MUDASIR \& AHMAD}

4. If $\theta=0, \lambda=\beta=1$ in (5), the inverse exponential distribution is obtained with the probability density function given as

$$
\mathrm{f}(x)=\frac{\alpha}{x^{2}} \exp \left(-\frac{\alpha}{x}\right), \quad x>0 ; \alpha>0
$$

\section{Limits of WGIWD}

1. $\operatorname{limf}_{x \rightarrow 0}(x)=\lim _{x \rightarrow 0}\left(\beta \alpha^{\beta-\theta} \lambda^{1-\frac{\theta}{\beta}} x^{\theta-\beta-1} \exp \left(-\lambda\left(\frac{\alpha}{x}\right)^{\beta}\right) / \Gamma\left(1-\frac{\theta}{\beta}\right)\right)$

$$
=\frac{\beta \alpha^{\beta-\theta} \lambda^{1-\frac{\theta}{\beta}}}{\Gamma\left(1-\frac{\theta}{\beta}\right)} \lim _{x \rightarrow 0} x^{\theta-\beta-1} \exp \left(-\lambda\left(\frac{\alpha}{x}\right)^{\beta}\right)=0
$$

2. $\operatorname{limf}_{x \rightarrow \infty}(x)=\lim _{x \rightarrow \infty}\left(\beta \alpha^{\beta-\theta} \lambda^{1-\frac{\theta}{\beta}} x^{\theta-\beta-1} \exp \left(-\lambda\left(\frac{\alpha}{x}\right)^{\beta}\right) / \Gamma\left(1-\frac{\theta}{\beta}\right)\right)$

$$
=\frac{\beta \alpha^{\beta-\theta} \lambda^{1-\frac{\theta}{\beta}}}{\Gamma\left(1-\frac{\theta}{\beta}\right)} \lim _{x \rightarrow \infty} x^{\theta-\beta-1} \exp \left(-\lambda\left(\frac{\alpha}{x}\right)^{\beta}\right)=\infty
$$

\section{Mode of WGIWD}

The mode of WGIWD is obtained by finding the first derivative of $\log \left(f_{w}(x)\right)$ with respect to $x$ and equating to zero, i.e.

$$
\frac{d}{d x} \log \left(\mathrm{f}_{w}(x)\right)=0
$$

Therefore, the mode at $x=x_{0}$ is given by 
WEIGHTED GENERALIZED INVERSE WEIBULL DISTRIBUTION

$$
\begin{aligned}
& \frac{d}{d x} \log \left(\frac{\beta \alpha^{\beta-\theta} \lambda^{1-\frac{\theta}{\beta}} x^{\theta-\beta-1} \exp \left(-\lambda\left(\frac{\alpha}{x}\right)^{\beta}\right)}{\Gamma\left(1-\frac{\theta}{\beta}\right)}\right)=0 \\
& \Rightarrow x^{\beta}=\frac{\beta \lambda \alpha^{\beta}}{\beta-\theta+1} \\
& \Rightarrow x_{0}=\alpha\left(\frac{\beta \lambda}{\beta-\theta+1}\right)^{\frac{1}{\beta}}
\end{aligned}
$$

\section{Moments and Moment Generating Function of WGIWD}

The $r^{\text {th }}$ moment about origin and the moment generating function are derived as follows:

Theorem 1. If a random variable $X$ has the weighted generalized inverse Weibull distribution, then the $r^{\text {th }}$ moment about origin is

$$
\mu_{r}^{\prime}=\left(\alpha \lambda^{\frac{1}{\beta}}\right)^{r} \frac{\Gamma\left(1-\frac{\theta+r}{\beta}\right)}{\Gamma\left(1-\frac{\theta}{\beta}\right)} .
$$

Proof. The $r^{\text {th }}$ moment of weighted generalized inverse Weibull distribution about the origin is obtained as

$$
\mu_{r}^{\prime}=\int_{0}^{\infty} x^{r} \mathrm{f}_{w}(x) d x .
$$

Using equation (5) in equation (6),

$$
\mu_{r}^{\prime}=\frac{\beta \alpha^{\beta-\theta} \lambda^{1-\frac{\theta}{\beta}}}{\Gamma\left(1-\frac{\theta}{\beta}\right)} \int_{0}^{\infty} x^{r+\theta-\beta-1} \exp \left(-\lambda\left(\frac{\alpha}{x}\right)^{\beta}\right) d x
$$




\section{MUDASIR \& AHMAD}

Put

$$
\begin{aligned}
& y=\lambda\left(\frac{\alpha}{x}\right)^{\beta} \\
& \Rightarrow x=\frac{\alpha \lambda^{\frac{1}{\beta}}}{y^{\frac{1}{\beta}}} \\
& \Rightarrow d x=-\frac{d y}{\beta \lambda \alpha^{\beta} x^{-\beta-1}}
\end{aligned}
$$

As $x \rightarrow 0, y \rightarrow \infty$ and as $x \rightarrow \infty, y \rightarrow 0$,

$$
\begin{aligned}
\mu_{r}^{\prime} & =\frac{\alpha^{r} \lambda^{\frac{r}{\beta}}}{\Gamma\left(1-\frac{\theta}{\beta}\right)} \int_{0}^{\infty} y^{\frac{-r-\theta}{\beta}} \exp (-y) d y \\
& \Rightarrow \mu_{r}^{\prime}=\left(\alpha \lambda^{\frac{1}{\beta}}\right)^{r} \frac{\Gamma\left(1-\frac{\theta+r}{\beta}\right)}{\Gamma\left(1-\frac{\theta}{\beta}\right)}
\end{aligned}
$$

This proves the theorem.

Corollary. If $r=1$ is inserted in equation (7), the mean of the WGIWD is obtained, which is given by

$$
\mu_{1}^{\prime}=\alpha \lambda^{\frac{1}{\beta}} \frac{\rho_{\theta+1}}{\rho_{\theta}}
$$

If $r=2$ in equation (7),

$$
\mu_{2}^{\prime}=\alpha^{2} \lambda^{\frac{2}{\beta}} \frac{\rho_{\theta+2}}{\rho_{\theta}}
$$

The variance is given by 


\section{WEIGHTED GENERALIZED INVERSE WEIBULL DISTRIBUTION}

$$
\mu_{2}=\frac{\alpha^{2} \lambda^{\frac{2}{\beta}}}{\rho_{\theta}}\left(\rho_{\theta+2}-\frac{\rho_{\theta+1}^{2}}{\rho_{\theta}}\right)=\sigma^{2} .
$$

If $r=3$ in equation (7),

$$
\begin{gathered}
\mu_{3}^{\prime}=\alpha^{3} \lambda^{\frac{3}{\beta}} \frac{\rho_{\theta+3}}{\rho_{\theta}}, \\
\mu_{3}=\mu_{3}^{\prime}-3 \mu_{2}^{\prime} \mu_{1}^{\prime}+2 \mu_{1}^{\prime 3} \\
\Rightarrow \mu_{3}=\frac{\alpha^{3} \lambda^{\frac{3}{\beta}}}{\rho_{\theta}^{3}}\left(\rho_{\theta}^{2} \rho_{\theta+3}-3 \rho_{\theta} \rho_{\theta+1} \rho_{\theta+2}+2 \rho_{\theta+1}^{3}\right)
\end{gathered}
$$

If $r=4$ in equation (7),

$$
\begin{gathered}
\mu_{4}^{\prime}=\alpha^{4} \lambda^{\frac{4}{\beta}} \frac{\rho_{\theta+4}}{\rho_{\theta}}, \\
\mu_{4}=\mu_{4}^{\prime}-4 \mu_{3}^{\prime} \mu_{1}^{\prime}+6 \mu_{2}^{\prime} \mu_{1}^{\prime 2}-3 \mu_{1}^{\prime 4} \\
\Rightarrow \mu_{4}=\frac{\alpha^{4} \lambda^{\frac{4}{\beta}}}{\rho_{\theta}^{4}}\left(\rho_{\theta}^{3} \rho_{\theta+4}-4 \rho_{\theta}^{2} \rho_{\theta+1} \rho_{\theta+3}+6 \rho_{\theta} \rho_{\theta+1}^{2} \rho_{\theta+2}-3 \rho_{\theta+1}^{4}\right)
\end{gathered}
$$

where

$$
\rho_{s}=\Gamma\left(1-\frac{s}{\beta}\right)
$$

Theorem 2. If a random variable $X$ follows the weighted generalized inverse Weibull distribution, then the moment generating function, denoted by $\mathrm{M}_{X}(t)$, is given by

$$
\mathrm{M}_{X}(t)=\sum_{r=0}^{\infty} \frac{t^{r}}{r !}\left(\alpha \lambda^{\frac{1}{\beta}}\right)^{r} \frac{\Gamma\left(1-\frac{\theta+r}{\beta}\right)}{\Gamma\left(1-\frac{\theta}{\beta}\right)} .
$$


Proof. By definition,

$$
\begin{aligned}
\mathrm{M}_{X}(t) & =\mathrm{E}\left(e^{t x}\right)=\int_{0}^{\infty} \mathrm{e}^{t x} \mathrm{f}_{w}(x) d x \\
& \Rightarrow \mathrm{M}_{X}(t)=\int_{0}^{\infty} \sum_{r=0}^{\infty} \frac{(t x)^{r}}{r !} \mathrm{f}_{w}(x) d x \\
& \Rightarrow \mathrm{M}_{X}(t)=\sum_{r=0}^{\infty} \frac{(t)^{r}}{r !} \mu_{r}^{\prime} \\
& \Rightarrow \mathrm{M}_{X}(t)=\sum_{r=0}^{\infty} \frac{t^{r}}{r !}\left(\alpha \lambda^{\frac{1}{\beta}}\right)^{r} \frac{\Gamma\left(1-\frac{\theta+r}{\beta}\right)}{\Gamma\left(1-\frac{\theta}{\beta}\right)}
\end{aligned}
$$

This proves the theorem.

\section{Standard Deviation and Coefficient of Variation}

By using the value of equation (10),

$$
\sigma=\alpha \lambda^{\frac{1}{\beta}} \sqrt{\frac{1}{\rho_{\theta}}\left(\rho_{\theta+2}-\frac{\rho_{\theta+1}^{2}}{\rho_{\theta}}\right)} .
$$

The coefficient of variation is defined as the ratio of the standard deviation to the mean. Usually, it is denoted by $C . V$ and is given by

$$
C . V=\frac{\sigma}{\mu} .
$$

By using the value of equations (15) and (8) in equation (16),

$$
C . V=\frac{\sqrt{\left(\rho_{\theta} \rho_{\theta+2}-\rho_{\theta+1}^{2}\right)}}{\rho_{\theta+1}} .
$$




\section{WEIGHTED GENERALIZED INVERSE WEIBULL DISTRIBUTION}

\section{Skewness and Kurtosis}

Skewness in terms of moments of frequency distribution is given by

$$
\beta_{1}=\frac{\mu_{3}^{2}}{\mu_{2}^{3}} .
$$

By substituting the value of equations (10) and (12) in equation (17),

$$
\begin{gathered}
\beta_{1}=\frac{\left(\rho_{\theta}^{2} \rho_{\theta+3}-3 \rho_{\theta} \rho_{\theta+1} \rho_{\theta+2}+2 \rho_{\theta+1}^{3}\right)^{2}}{\left(\rho_{\theta} \rho_{\theta+2}-\rho_{\theta+1}^{2}\right)^{3}}, \\
\gamma_{1}=\sqrt{\beta_{1}} \\
\Rightarrow \gamma_{1}=\frac{\left(\rho_{\theta}^{2} \rho_{\theta+3}-3 \rho_{\theta} \rho_{\theta+1} \rho_{\theta+2}+2 \rho_{\theta+1}^{3}\right)}{\left(\rho_{\theta} \rho_{\theta+2}-\rho_{\theta+1}^{2}\right)^{\frac{3}{2}}}
\end{gathered}
$$

Kurtosis is given by

$$
\beta_{2}=\frac{\mu_{4}}{\mu_{2}^{2}}
$$

By substituting the value of equations (10) and (14) in equation (18),

$$
\begin{aligned}
& \beta_{2}=\frac{\rho_{\theta}^{3} \rho_{\theta+4}-4 \rho_{\theta}^{2} \rho_{\theta+1} \rho_{\theta+3}+6 \rho_{\theta} \rho_{\theta+1}^{2} \rho_{\theta+2}-3 \rho_{\theta+1}^{4}}{\left(\rho_{\theta} \rho_{\theta+2}-\rho_{\theta+1}^{2}\right)^{2}} \\
\gamma_{2}= & \beta_{2}-3 \\
\Rightarrow & \gamma_{2}=\frac{\rho_{\theta}^{3} \rho_{\theta+4}-4 \rho_{\theta}^{2} \rho_{\theta+1} \rho_{\theta+3}+6 \rho_{\theta} \rho_{\theta+1}^{2} \rho_{\theta+2}-3 \rho_{\theta+1}^{4}}{\left(\rho_{\theta} \rho_{\theta+2}-\rho_{\theta+1}^{2}\right)^{2}}-3
\end{aligned}
$$




\section{MUDASIR \& AHMAD}

Table 1. Mean, variance, mode, harmonic mean (HM), standard deviation (STD), coefficient of variation (CV), coefficient of skewness (CS), and coefficient of kurtosis (CK)

\begin{tabular}{rrrrrrrrrrrr}
$\boldsymbol{\alpha}$ & $\boldsymbol{\beta}$ & $\boldsymbol{\lambda}$ & $\boldsymbol{\theta}$ & Mean & Variance & Mode & HM & STD & CV & CS & CK \\
\hline 1 & 5 & 1 & 0 & 1.16423 & 0.13376 & 0.96419 & 0.85893 & 0.36573 & 0.31414 & 3.53507 & 48.09151 \\
2 & 12 & 5 & 1 & 2.44575 & 4.92882 & 2.28705 & 2.13866 & 2.22009 & 0.90773 & 1.87428 & 10.48306 \\
3 & 13 & 6 & 2 & 3.70396 & 12.76274 & 3.46459 & 3.20104 & 3.57249 & 0.96450 & 1.92437 & 10.83551 \\
4 & 14 & 7 & 3 & 4.96756 & 23.85802 & 4.64735 & 4.25309 & 4.88446 & 0.98327 & 1.97100 & 11.16541 \\
5 & 15 & 8 & 4 & 6.23065 & 38.17854 & 5.82957 & 5.29441 & 6.17887 & 0.99168 & 2.01433 & 11.47340 \\
7 & 16 & 10 & 5 & 8.79611 & 77.22640 & 8.23013 & 7.42856 & 8.78785 & 0.99906 & 2.05455 & 11.76039 \\
9 & 17 & 13 & 6 & 11.41719 & 130.87461 & 10.68234 & 9.59351 & 11.44004 & 1.00200 & 2.09187 & 12.02751 \\
11 & 18 & 14 & 7 & 13.92412 & 195.18381 & 13.02714 & 11.65105 & 13.97082 & 1.00335 & 2.12650 & 12.27595 \\
13 & 20 & 16 & 8 & 16.20590 & 264.23568 & 15.25821 & 13.76022 & 16.25532 & 1.00304 & 2.06380 & 11.57867 \\
15 & 22 & 18 & 9 & 18.44890 & 342.21604 & 17.46109 & 15.86089 & 18.49908 & 1.00271 & 2.01321 & 11.04287 \\
\hline
\end{tabular}

\section{Harmonic Mean of WGIWD}

The harmonic mean is usually denoted by $H$ and is given as

$$
\begin{aligned}
\frac{1}{H} & =\int_{0}^{\infty} \frac{1}{x} \mathrm{f}_{w}(x) d x \\
& \Rightarrow H=\alpha \lambda^{\frac{1}{\beta}} \frac{\Gamma\left(1-\frac{\theta}{\beta}\right)}{\Gamma\left(1-\frac{\theta-1}{\beta}\right)}
\end{aligned}
$$

\section{Shannon's Entropy of the WGIWD}

The concept of Shannon's entropy is the central role of information theory. For a random variable entropy is defined in terms of its probability distribution and is a good measure of uncertainty. If $X$ is a non-negative continuous random variable with the probability density function $\mathrm{f}(x)$, then the Shannon's entropy of $X$ is defined as

$$
\begin{aligned}
\mathrm{H}(\mathrm{f}(x)) & =-\int_{-\infty}^{\infty} \mathrm{f}(x) \log \mathrm{f}(x) d x \\
& =\mathrm{E}(-\log (\mathrm{f}(x)))
\end{aligned}
$$

provided the integral exists. 


\section{WEIGHTED GENERALIZED INVERSE WEIBULL DISTRIBUTION}

Theorem 3. If the random variable $X$ has a weighted generalized inverse Weibull distribution with the probability density function defined in (5), then the Shannon's entropy of WGIWD is

$$
\mathrm{H}\left(\mathrm{f}_{w}(x)\right)=\frac{1}{\beta} \log \lambda+\log \left(\frac{\alpha}{\beta}\right)+\log \left(\Gamma\left(1-\frac{\theta}{\beta}\right)\right)+\left(\frac{\theta-\beta-1}{\beta}\right) \psi\left(1-\frac{\theta}{\beta}\right)-\frac{\theta}{\beta}+1
$$

where

$$
\psi(\theta+\lambda)=\frac{\Gamma^{\prime}\left(1-\frac{\theta}{\beta}\right)}{\Gamma\left(1-\frac{\theta}{\beta}\right)} .
$$

Proof. Shannon's entropy is defined as

$$
\begin{aligned}
\mathrm{H}\left(\mathrm{f}_{w}(x)\right) & =\mathrm{E}\left(-\log \left(\mathrm{f}_{w}(x)\right)\right) \\
& =\mathrm{E}\left(-\log \left(\frac{\beta \alpha^{\beta-\theta} \lambda^{1-\frac{\theta}{\beta}} x^{\theta-\beta-1} \exp \left(-\lambda\left(\frac{\alpha}{x}\right)^{\beta}\right)}{\Gamma\left(1-\frac{\theta}{\beta}\right)}\right)\right) \\
& =-\log \left(\frac{\beta \alpha^{\beta-\theta} \lambda^{1-\frac{\theta}{\beta}}}{\Gamma\left(1-\frac{\theta}{\beta}\right)}\right)-(\theta-\beta-1) \mathrm{E}(\log x)+\lambda \alpha^{\beta} \mathrm{E}\left(x^{-\beta}\right)
\end{aligned}
$$

From equation (7),

$$
\begin{aligned}
& \mathrm{E}\left(x^{-\beta}\right)=\left(\alpha \lambda^{\frac{1}{\beta}}\right)^{-\beta}\left(1-\frac{\theta}{\beta}\right) . \\
& \mathrm{E}(\log x)=\int_{0}^{\infty} \log (x) \mathrm{f}_{w}(x) d x
\end{aligned}
$$




\section{MUDASIR \& AHMAD}

$$
=\frac{\beta \alpha^{\beta-\theta} \lambda^{1-\frac{\theta}{\beta}}}{\Gamma\left(1-\frac{\theta}{\beta}\right)} \int_{0}^{\infty} \log (x) x^{\theta-\beta-1} \exp \left(-\lambda\left(\frac{\alpha}{x}\right)^{\beta}\right) d x
$$

By substituting $y=\lambda(\alpha / x)^{\beta}$,

$$
\mathrm{E}(\log x)=\frac{1}{\beta} \log \lambda+\log (\alpha)-\left(\frac{1}{\beta}\right) \psi\left(1-\frac{\theta}{\beta}\right)
$$

Substituting the value of equations (21), (22) in equation (20),

$$
\begin{aligned}
& \mathrm{H}\left(\mathrm{f}_{w}(x)\right)=-\log \left(\frac{\beta \alpha^{\beta-\theta} \lambda^{1-\frac{\theta}{\beta}}}{\Gamma\left(1-\frac{\theta}{\beta}\right)}\right)-(\theta-\beta-1)\left(\frac{1}{\beta} \log \lambda+\log (\alpha)-\left(\frac{1}{\beta}\right) \psi\left(1-\frac{\theta}{\beta}\right)\right) \\
&+\lambda \alpha^{\beta}\left(\alpha \lambda^{\frac{1}{\beta}}\right)^{-\beta}\left(1-\frac{\theta}{\beta}\right) \\
&=\frac{1}{\beta} \log \lambda+\log \left(\frac{\alpha}{\beta}\right)+\log \left(\Gamma\left(1-\frac{\theta}{\beta}\right)\right)+\left(\frac{\theta-\beta-1}{\beta}\right) \psi\left(1-\frac{\theta}{\beta}\right)-\frac{\theta}{\beta}+1
\end{aligned}
$$

This proves the theorem.

\section{Entropy Estimation}

The probability density function of weighted generalized inverse Weibull distribution is

$$
\mathrm{f}_{w}(x)=\frac{\beta \alpha^{\beta-\theta} \lambda^{1-\frac{\theta}{\beta}} x^{\theta-\beta-1} \exp \left(-\lambda\left(\frac{\alpha}{x}\right)^{\beta}\right)}{\Gamma\left(1-\frac{\theta}{\beta}\right)}, \quad x>0 ; \alpha, \beta, \theta, \lambda>0 .
$$




$$
\begin{aligned}
& \log (L)=n \log \left(\frac{\beta \alpha^{\beta-\theta} \lambda^{1-\frac{\theta}{\beta}}}{\Gamma\left(1-\frac{\theta}{\beta}\right)}\right)+(\theta-\beta-1) \sum_{i=1}^{n} x_{i}-\lambda \alpha^{\beta} \sum_{i=1}^{n} x_{i}^{-\beta} \\
& \Rightarrow-\frac{\log (L)}{n}=-\log \left(\frac{\beta \alpha^{\beta-\theta} \lambda^{1-\frac{\theta}{\beta}}}{\Gamma\left(1-\frac{\theta}{\beta}\right)}\right)-(\theta-\beta-1) \mathrm{E}(\log x)+\lambda \alpha^{\beta} \mathrm{E}\left(x^{-\beta}\right)
\end{aligned}
$$

On comparing equations (20) and (23),

$$
\hat{\mathrm{H}}\left(\mathrm{f}_{w}(x)\right)=-\frac{\log (L)}{n} \text {. }
$$

\section{Akaike and Bayesian Information Criterion}

For comparing different models, use the concept of Akaike information criterion (AIC) and Bayesian information criterion (BIC). The model which has the smallest values of AIC and BIC is considered the best model. Suppose there is a statistical model of some data set. Let $L$ be the maximized value of the likelihood function for the model. Let $K$ be the number of estimated parameters in the model. Then AIC and BIC of the model are given by

$$
\begin{gathered}
\mathrm{AIC}=2 K-2 \log (L), \\
\mathrm{BIC}=K \log (n)-2 \log (L),
\end{gathered}
$$

where $n$ is the number of observations or equivalently the sample size.

From equation (24),

$$
\log (L)=-n \hat{\mathrm{H}}\left(\mathrm{f}_{w}(x)\right)
$$

Then, for the weighted generalized inverse Weibull distribution, 


$$
\begin{aligned}
& \mathrm{AIC}=2 K+2 n \hat{\mathrm{H}}\left(\mathrm{f}_{w}(x)\right) \\
& \mathrm{BIC}=K \log (n)+2 n \hat{\mathrm{H}}\left(\mathrm{f}_{w}(x)\right)
\end{aligned}
$$

\section{Example: Real-Life Data}

Consider a real-life data set to establish the superiority of WGIWD over other distributions. The data set, given by Lee and Wang (2003), represents remission times (in months) of a random sample of 128 bladder cancer patients. For establishing the superiority of WGIWD, the calculated values of AIC and BIC are presented in Table 2 for the following data: $0.08,2.09,3.48,4.87,6.94$, 8.66, 13.11, $23.63,0.20,2.23,3.52,4.98,6.97,9.02,13.29,0.40,2.26,3.57,5.06,7.09,9.22$, $13.80,25.74,0.50,2.46,3.64,5.09,7.26,9.47,14.24,25.82,0.51,2.54,3.70,5.17$, 7.28, 9.74, 14.76, 26.31, 0.81, 2.62, 3.82, 5.32, 7.32, 10.06, 14.77, 32.15, 2.64, 3.88, $5.32,7.39,10.34,14.83,34.26,0.90,2.69,4.18,5.34,7.59,10.66,15.96,36.66$, $1.05,2.69,4.23,5.41,7.62,10.75,16.62,43.01,1.19,2.75,4.26,5.41,7.63,17.12$, $46.12,1.26,2.83,4.33,5.49,7.66,11.25,17.14,79.05,1.35,2.87,5.62,7.87,11.64$, $17.36,1.40,3.02,4.34,5.71,7.93,11.79,18.10,1.46,4.40,5.85,8.26,11.98,19.13$, $1.76,3.25,4.50,6.25,8.37,12.02,2.02,3.31,4.51,6.54,8.53,12.03,20.28,2.02$, $3.36,6.76,12.07,21.73,2.07,3.36,6.93,8.65,12.63,22.69$.

From Table 2, the weighted generalized inverse Weibull distribution has the smallest AIC and BIC values as compared to other models. It may be concluded the weighted generalized inverse Weibull distribution provided the best fit to this specific data set. The histogram for the above data set and estimated densities of WGIWD, LGIWD, GIWD, IWD and IED have been plotted in Figure 5.

Table 2. AIC, BIC for different models

\begin{tabular}{rrrrrrrrr} 
& \multicolumn{9}{c}{ Estimates } & \multicolumn{2}{c}{$\begin{array}{c}\text { Shannon's } \\
\text { Model }\end{array}$} & Alpha & Beta & Lambda & Theta & -2log I & AIC & BIC \\
\hline WGIWD & 1.0522 & 0.7767 & 1.8851 & 0.0929 & 888.3770 & 3.4700 & 896.3770 & 896.8060 \\
LGIWD & 2.5031 & 1.4185 & 0.1410 & - & 957.4330 & 3.7400 & 963.4330 & 963.7550 \\
GIWD & 0.0738 & 0.8409 & 22.0745 & - & 891.7850 & 3.4830 & 897.7850 & 904.4280 \\
IWD & 4.9649 & 0.6894 & - & - & 898.5740 & 3.5100 & 902.5740 & 907.0020 \\
IED & 2.4847 & - & - & - & 920.7650 & 3.5970 & 922.7650 & 924.9760 \\
\hline
\end{tabular}




\section{WEIGHTED GENERALIZED INVERSE WEIBULL DISTRIBUTION}

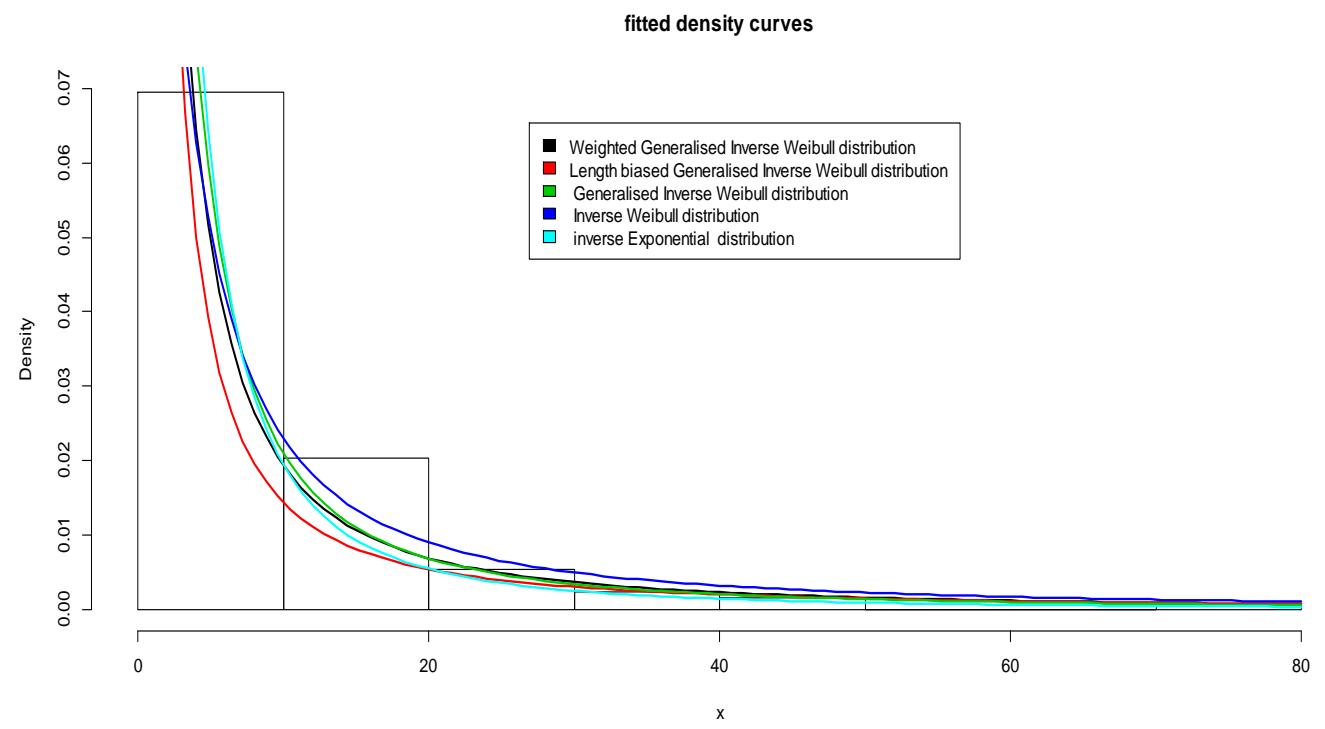

Figure 5. Plots for the histogram and estimated densities

\section{Conclusion}

The weighted version of generalized inverse Weibull distribution, known as weighted generalized inverse Weibull distribution (WGIWD), was introduced. The new model was compared with other sub-models by using real life data set and it was concluded the new model provides the best fit for a specific data set as compared with other models.

\section{References}

Calabria, R., \& Pulcini, G. (1994). Bayesian 2-sample prediction for the inverse Weibull distribution. Communications in Statistics - Theory and Methods, 23(1994), 1811-1824. doi: 10.1080/03610929408831356

de Gusmão, F. R. S., Ortega, E. M. M., \& Cordeiro, G. M. (2009). The generalized inverse Weibull distribution. Statistical Papers, 52(3), 591-619. doi: 10.1007/s00362-009-0271-3

Fatima, K., \& Ahmad, S. P. (2017). Weighted inverse Rayleigh distribution. International Journal of Statistics and Systems, 12(1), 119-137. Retrieved from https://www.ripublication.com/ijss17/ijssv12n1_10.pdf 


\section{MUDASIR \& AHMAD}

Fisher, R. A. (1934). The effects of methods of ascertainment upon the estimation of frequencies. Annals of Eugenics, 6(1), 13-25. doi: 10.1111/j.14691809.1934.tb02105.x

Gupta, R. C., \& Keating, J. P. (1985). Relations for reliability measures under length biased sampling. Scandinavian Journal of Statistics, 13(1), 49-56. Available from https://www.jstor.org/stable/4616010

Gupta, R. C., \& Kirmani, S. N. U. A. (1990). The role of weighted distributions in stochastic modeling. Communication Statistics - Theory and Methods, 19(9), 3147-3162. doi: 10.1080/03610929008830371

Jan, U., Fatima, K., \& Ahmad, S. P. (2017). On weighted Ailamujia distribution and its applications to lifetime data. Journal of Statistics Applications \& Probability, 6(3), 619-633. doi: 10.18576/jsap/060317

Kersey, J. X. (2010). Weighted inverse Weibull and beta-inverse Weibull distribution (Unpublished Master's thesis). Georgia Southern University, Statesboro, GA. Retrieved from https://digitalcommons.georgiasouthern.edu/etd/661/

Kundu, D., \& Howlader, H. (2010). Bayesian inference and prediction of the inverse Weibull distribution for Type-II censored data. Computational Statistics \& Data Analysis, 54(6), 1547-1558. doi: 10.1016/j.csda.2010.01.003

Mudasir, S., \& Ahmad, S. P. (2015). Structural properties of length biased Nakagami distribution. International Journal of Modern Mathematical Sciences, 13(3), 217-227.

Mudasir, S., Ahmed, A., \& Ahmad, S. P. (2015). A note on Bayesian estimation of inverse Weibull distribution under LINEX and quadratic loss functions. International Journal of Modern Mathematical Sciences, 13(2), 170177.

Naqash, S., Ahmad, S. P., \& Ahmed, A. (2016). Bayesian analysis of generalized inverse Weibull distribution. International Journal of Modern Mathematical Sciences, 14(2), 183-196.

Oluyede, B. O. (1999). On inequalities and selection of experiments for length-biased distributions. Probability in the Engineering and Informational Sciences, 13(2), 169-185. doi: 10.1017/s0269964899132030

Patil, G. P., \& Rao, C. R. (1978). Weighted distributions and size-biased sampling with applications to wildlife populations and human families.

Biometrics, 34(2), 179-184. 10.2307/2530008 


\section{WEIGHTED GENERALIZED INVERSE WEIBULL DISTRIBUTION}

Rao, C. R. (1965). On discrete distributions arising out of methods of ascertainment, in classical and contagious discrete distributions. In G. P. Patil (Ed.), Classical and contagious discrete distributions: Proceedings of the international symposium (pp. 320-332). Calcutta, India: Pergamon Press and Statistical Publishing Society.

Shaban, S. A. \& Boudrissa, N. A. (2007). The Weibull length biased distribution: Properties and estimation. InterStat, 2007(January). Retrieved from http://interstat.statjournals.net/YEAR/2007/abstracts/0701002.php 